\title{
A prospective observational study of bacteraemia in adults admitted to an urban Mozambican hospital
} \author{
R T Schooley, ${ }^{1} \mathrm{MD}$; E Aronoff-Spencer, ${ }^{1} \mathrm{MD}, \mathrm{PhD}$ \\ ${ }^{1}$ University of California, San Diego, USA \\ ${ }^{2}$ Universidade Eduardo Mondlane, Maputo, Mozambique \\ ${ }^{3}$ Emory University, Atlanta, GA, USA \\ ${ }^{4}$ University of Iowa, Iowa City, USA \\ ${ }^{5}$ National Institutes of Health, Bethesda, MD, USA
}

M Preziosi, ${ }^{1}$ MD; T F Zimba, ${ }^{2} \mathrm{MD}$; K Lee, ${ }^{3} \mathrm{MD} ; \mathbf{M}$ Tomas, ${ }^{2} \mathrm{MD}$; S Kinlin, ${ }^{2} \mathrm{MD}$; C Nhatave-Paiva, ${ }^{2} \mathrm{MD} ; \mathbf{R}$ Bene, ${ }^{2} \mathrm{MD}$; T Paunde, ${ }^{2} \mathrm{MD}$; H Lopes, ${ }^{2} \mathrm{MD}$; S Kalkhoff, ${ }^{4} \mathrm{MD}$; V Prathap, ${ }^{1} \mathrm{MD}$; K Akrami, ${ }^{5} \mathrm{MD}$; E V Noormahomed ${ }^{1,2} \mathrm{MD}, \mathrm{PhD}$;

Corresponding author: M Preziosi (mpreziosi@ucsd.edu)

Background. Bacteraemia is a common cause of fever among patients presenting to hospitals in sub-Saharan Africa. The worldwide rise of antibiotic resistance makes empirical therapy increasingly difficult, especially in resource-limited settings.

Objectives. To describe the incidence of bacteraemia in febrile adults presenting to Maputo Central Hospital (MCH), an urban referral hospital in the capital of Mozambique, and characterise the causative organisms and antibiotic susceptibilities. We aimed to describe the antibiotic prescribing habits of local doctors, to identify areas for quality improvement.

Methods. Inclusion criteria were: ( $i) \geq 18$ years of age; (ii) axillary temperature $\geq 38^{\circ} \mathrm{C}$ or $\leq 35^{\circ} \mathrm{C}$; (iii) admission to $\mathrm{MCH}$ medical wards in the past 24 hours; and (iv) no receipt of antibiotics as an inpatient. Blood cultures were drawn from enrolled patients and incubated using the BacT/Alert automated system (bioMérieux, France). Antibiotic susceptibilities were tested using the Kirby-Bauer disc diffusion method. Results. Of the 841 patients enrolled, 63 (7.5\%) had a bloodstream infection. The most common isolates were Staphylococcus aureus, Escherichia coli, and non-typhoidal Salmonella. Antibiotic resistance was common, with 20/59 (33.9\%) of all bacterial isolates showing resistance to ceftriaxone, the broadest-spectrum antibiotic commonly available at MCH. Receipt of insufficiently broad empirical antibiotics was associated with poor in-hospital outcomes (odds ratio $8.05 ; 95 \%$ confidence interval $1.62-39.91 ; p=0.04$ ).

Conclusion. This study highlights several opportunities for quality improvement, including educating doctors to have a higher index of suspicion for bacteraemia, improving local antibiotic guidelines, improving communication between laboratory and doctors, and increasing the supply of some key antibiotics.

S Afr Med J 2015;105(5):370-374. DOI:10.7196/SAMJ.8780

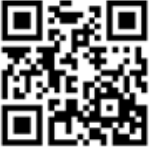

Bacteraemia is a common cause of fever among patients presenting to hospitals in sub-Saharan Africa (SSA), but it remains underdiagnosed because many facilities lack the proper resources, and it often presents in nonspecific ways. ${ }^{[1]}$ Furthermore, the worldwide rise of antibiotic resistance makes empirical therapy increasingly difficult, especially in resource-limited settings..$^{[2,3]}$ Maputo Central Hospital (MCH) is a 1200 -bed national referral hospital in Maputo, the capital of Mozambique. It is the principal teaching hospital in Mozambique, in collaboration with Universidade Eduardo Mondlane (UEM). MCH has a modern, automated system in place to perform blood cultures and diagnose bacteraemia, yet historically the system was underutilised. We designed a prospective study to determine the incidence of bacteraemia in patients presenting with fever, a common presenting sign in the medical wards of $\mathrm{MCH}$. The majority of patients admitted to the medical wards at $\mathrm{MCH}$ are HIV-positive, commonly presenting in late stages of disease, and not on antiretroviral therapy. This is consistent with other reports from Mozambique and the greater region. ${ }^{[4,5]}$ The differential diagnosis for fever is broad in this population and frequently includes bacteraemia, ${ }^{[1,6-8]}$ although it was rarely considered at $\mathrm{MCH}$ prior to this study.

The greater context of this study is the Medical Education Partnership Initiative between the University of California, San Diego, USA, and UEM, which focuses on strengthening the educational and research infrastructure of Mozambique's three public medical schools. A major priority of this collaboration is to recruit trainees and junior faculty into lifelong careers in medical education and research while providing them with the skills to make these careers sustainable. ${ }^{[9,10]}$ At the outset of the collaboration, doctors working in the internal medicine department at $\mathrm{MCH}$ were surveyed in order to identify problems considered to be of highest importance. 'We are blind' was a specific phrase repeated by numerous doctors in this survey, in reference not only to their limited diagnostic resources but also to the lack of medical research done locally. Early efforts to address these problems included implementation of a regular schedule of clinical conferences and journal clubs in the residency training programme, accompanied by improved access to internet and medical information in the hospital, in order to establish a culture of scientific enquiry and evidence-based medicine. Focus later shifted to improvement of biomedical informatics, as well as infrastructure for clinical, operational and epidemiological research. Opportunities for Mozambican medical residents to participate in research projects were sought. This study is the first to be carried out in part by residents in the internal medicine training programme at $\mathrm{MCH}$. It also represents the first study of bacteraemia in a hospitalised adult Mozambican population.

In addition to describing the incidence of bacteraemia in febrile adults presenting to $\mathrm{MCH}$ and creating an antibiogram, we set out 
to describe the antibiotic prescribing habits of local doctors and to identify links with patient outcomes, with a view to identifying highyield areas for future quality improvement projects.

\section{Methods \\ Ethical statement}

Ethical approval was obtained specifically for this study from the University of California, San Diego Institutional Review Board as well as the Mozambican National Committee for Bioethics and Health.

\section{Study team}

We assembled a study team of local Mozambican healthcare workers: a nurse who enrolled and phlebotomised patients, five internal medicine residents who recorded patient clinical information and outcomes, a microbiology laboratory technician charged with processing blood cultures, and a senior infectious disease expert. In addition, there were two collaborators from the USA, one on-site and one off-site.

\section{Patient enrolment}

From September 2011 through October 2012, and then again from July 2013 through March 2014, the study nurse enrolled patients on weekday mornings from the internal medicine wards. Inclusion criteria were as follows: $(i) \geq 18$ years of age; (ii) axillary temperature $\geq 38^{\circ} \mathrm{C}$ or $\leq 35^{\circ} \mathrm{C}$; (iii) admission in the past 24 hours; and (iv) no receipt of antibiotics as an inpatient. Each patient gave written informed consent. If patients were unable to give consent, an attendant (usually a family member) consented for them. We initially attempted to enrol patients from the emergency room, which was frequently overcrowded and understaffed, but abandoned these efforts out of concern for patient safety and a desire to not influence emergency room doctors' decisions to admit patients. Once enrolled, the nurse drew $20 \mathrm{~mL}$ of blood from each patient and aseptically inoculated two BacT/Alert aerobic blood culture bottles (bioMérieux, France).

\section{Data collection}

The in-hospital medical team was responsible for all clinical decisions regarding patient management. Each patient was independently followed by one of the Mozambican internal medicine residents on the study team, who recorded laboratory data as well as outcomes 'improved', 'unimproved' or 'died' from patient charts. Members of the study team did not make clinical decisions but did inform the doctors caring for the patient that their patient had been enrolled in a study and facilitated communication of blood culture results from the laboratory. Nonetheless, in some cases patients were discharged before a complete data set had been recorded. Each team member received an Android tablet with a customised Health Information System application (keep.distributedhealth.org) for data entry. Data were synchronised automatically from tablets to a server when internet was available. An offsite US collaborator reviewed data on a weekly basis and debriefed the study team.

\section{Bacterial culture, identification and antibiotic susceptibility testing}

Blood cultures were processed using BacT/Alert i AST bottles in a BacT/ALERT 3D machine (bioMérieux). Isolates from positive cultures were identified by standard biochemical tests as set out in Clinical Laboratory Standards Institute (CLSI) guidelines. ${ }^{[1]}$ Cultures were considered contaminated if they grew coagulase-negative Staphylococcus or diptheroids. Antimicrobial susceptibility testing was performed by standard disc diffusion methods to trimethoprim/ sulfamethoxazole $(1.25 / 23.75 \mu \mathrm{g})$, chloramphenicol $(30 \mu \mathrm{g})$, oxacillin $(1 \mu \mathrm{g})$, penicillin $(10 \mu \mathrm{g})$, ampicillin $(10 \mu \mathrm{g})$, tetracycline $(30 \mu \mathrm{g})$, gentamicin $(10 \mu \mathrm{g})$ ceftriaxone $(30 \mu \mathrm{g})$, ciprofloxacin $(5 \mu \mathrm{g})$ and imipenem $(10 \mu \mathrm{g})$. Phenotypic methicillin-resistant Staphylococcus aureus (MRSA) were determined by oxacillin disc and cefoxitin disc and tested for resistance to vancomycin. In Salmonella isolates, antibiotic susceptibilities were also confirmed using the VitekII system (Card AB AST-N156, bioMérieux). Isolates flagged as extended-spectrum $\beta$-lactamase (ESBL) or demonstrating resistance to ceftriaxone or ceftazidime by the disc diffusion method were phenotypically confirmed by the combined double-disc method using ceftazidime and cefepime alone and in combination with clavulanic acid. Breakpoints for resistance were according to CLSI guidelines. ${ }^{[11]}$

\section{Clinical laboratory data}

Clinical laboratory data relevant to this study were processed in five different laboratories. For patients who did not have clearly documented HIV status, a rapid test was performed using both the Determine HIV1/2 Rapid Test (Abbot Laboratories, USA) and the Unigold HIV Rapid Test (Trinity Biotech, Ireland). Per hospital protocol, in the case of discordant results both tests were repeated in one month (there is no third test available as a tiebreaker at $\mathrm{MCH})$. CD4 counts were determined using a FACS-Calibur flow cytometer (BD Biosciences, USA). Complete blood counts were determined with an XE-2100 haematology analyser (Sysmex, Japan). Plasmodium falciparum malaria was diagnosed first with a rapid immunochromatographic test for the HRP-2 antigen (First Response, Premiere Medical Corporation, India); if positive, a Giemsa-stained thick and thin smear was analysed in the regular course of clinical duty by a locally trained microscopist paid by the Ministry of Health. In cases where sputum smears were ordered, Ziehl-Neelsen-stained slides were similarly interpreted by a hospital microscopist. Owing to limited resources, we were not able to implement quality control for microscopy-based diagnoses, and our data reflect what was available to Mozambican doctors charged with caring for enrolled patients.

\section{Statistical analysis}

Data were exported from the online database to an Excel spreadsheet. Descriptive statistics were calculated with Excel and further analysis was done with free online software (vassarstats.net). Categorical data were compared with the $\chi^{2}$ test and Fisher's exact test where appropriate. Odds ratios (ORs) and 95\% confidence intervals (CIs) were calculated for clinical predictors of bacteraemia, and clinical predictors for poor outcomes ('unimproved' or 'died') in patients with bacteraemia. In cases where specific clinical variables or outcomes were not available for all patients, only those patients for whom the variables were known were used in the analysis. Two-tailed $p$-values of $<0.05$ were considered statistically significant.

\section{Results}

Of the 841 patients enrolled, 63 (7.5\%) had a bloodstream infection. Of the 765 (91.1\%) patients with a documented outcome, 138 (18.0\%) died while in the hospital.

Thirteen of $56(23.2 \%)$ patients with bacteraemia and a documented outcome died while in the hospital, but bacteraemia was not a significant predictor of in-hospital mortality (OR 1.41; $95 \%$ CI $0.74-2.70 ; p=0.37$ ). However, bacteraemia was significantly linked to the combined endpoint of 'died' and 'unimproved' as a hospital outcome (OR 1.89; 95\% CI 1.07 - 3.39; $p=0.03$ ). Characteristics of the patient population and associations with 
bacteraemia are set out in Table 1 . There were very few factors associated with bacteraemia with a $p$-value $<0.20$, so a multifactorial analysis was not done.

Table 1. Characteristics of study population $(N=841)$ and predictors of bloodstream infection*

\begin{tabular}{|c|c|c|c|c|}
\hline \multirow[b]{2}{*}{ Characteristic } & \multicolumn{2}{|c|}{ Bloodstream infection } & \multirow[b]{2}{*}{ OR $(95 \% \mathrm{CI})$} & \multirow[b]{2}{*}{$p$-value } \\
\hline & Yes, $n(\%)$ & No, $n$ & & \\
\hline Male $(N=425)$ & $35(8.2)$ & 390 & $1.20(0.72-2.00)$ & 0.52 \\
\hline Female $(N=416)$ & $29(7.0)$ & 387 & $0.84(0.50-1.39)$ & 0.52 \\
\hline $\operatorname{SIRS}^{\dagger}(N=663)$ & $50(7.5)$ & 613 & $0.658(0.36-1.21)$ & 0.23 \\
\hline No SIRS $^{\dagger}(N=136)$ & $15(11.0)$ & 121 & $1.52(0.83-2.79)$ & 0.23 \\
\hline $\mathrm{SBP} \leq 90 \mathrm{mmHg}(N=90)$ & $12(13.3)$ & 78 & $1.84(0.94-3.56)$ & 0.06 \\
\hline $\mathrm{SBP}>90 \mathrm{mmHg}(N=658)$ & $53(8.1)$ & 635 & $0.54(0.28-1.06)$ & 0.06 \\
\hline $\mathrm{Hb}<5 \mathrm{mg} / \mathrm{dL}(N=98)$ & $18(18.4)$ & 80 & $1.78(0.98-3.23)$ & 0.06 \\
\hline $\mathrm{Hb} \geq 5 \mathrm{mg} / \mathrm{dL}(N=660)$ & $47(7.1)$ & 372 & $0.56(0.31-1.02)$ & 0.06 \\
\hline $\mathrm{HIV}+(N=652)$ & $55(8.4)$ & 597 & $1.07(0.53-2.16)$ & 1.0 \\
\hline HIV- $(N=126)$ & $10(7.9)$ & 116 & $0.94(0.46-1.89)$ & 1.0 \\
\hline CD4 $<200$ cells $/ \mu L^{\ddagger}(N=382)$ & $25(6.8)$ & 357 & $0.93(0.42-2.04)$ & 1.0 \\
\hline CD4 $>200$ cells $/ \mu L^{\ddagger}(N=128)$ & $9(7.0)$ & 119 & $1.08(0.49-2.38)$ & 1.0 \\
\hline $\mathrm{HIV}+$ on ARVs $(N=263)$ & $24(9.1)$ & 239 & $0.80(0.46-1.41)$ & 0.48 \\
\hline HIV+ not on ARVs $(N=279)$ & $31(11.1)$ & 248 & $1.24(0.71-2.18)$ & 0.48 \\
\hline $\begin{array}{l}\text { HIV+ on TMP-SMX } \\
\text { prophylaxis }(N=453)\end{array}$ & $34(7.5)$ & 419 & $0.69(0.39-1.22)$ & 0.22 \\
\hline $\begin{array}{l}\text { HIV+ without TMP-SMX } \\
\text { prophylaxis }(N=199)\end{array}$ & $21(10.5)$ & 178 & $1.45(0.82-2.57)$ & 0.22 \\
\hline \multicolumn{5}{|c|}{$\begin{array}{l}\text { SIRS }=\text { systemic inflammatory response syndrome; } \mathrm{SBP}=\text { systolic blood pressure; } \mathrm{Hb}=\text { haemoglobin; } \mathrm{ARVs}=\text { antiretrovirals; } \\
\text { TMP-SMX }=\text { trimethoprim-sulfamethoxazole. } \\
{ }^{*} \text { Owing to missing data, not all } n \text { 's in each category add up to total } N \text {. } \\
\text { 'SIRS defined as at least two of the following: axillary temperature } \geq 38^{\circ} \mathrm{C} \text {, heart rate }>90 \mathrm{bpm} \text {, respiratory rate }>20 / \mathrm{min} \text {, and } \\
\text { white cell count }<4 \times 10^{9} \text { cells } / \mathrm{L} \text { or }>11 \times 10^{9} \text { cells } / \mathrm{L} \text {. } \\
\text { w Data available for } 34 \text { of } 55 \mathrm{HIV}+\text { patients with bacteraemia. }\end{array}$} \\
\hline
\end{tabular}

Fifty blood cultures (5.9\%) were considered to be contaminants. The most common pathogenic isolates were $S$. aureus, E. coli and non-typhoidal Salmonella (NTS) (Table 2). Eight of 17 S. aureus isolates (47.0\%) were MRSA, consistent with local hospital epidemiology, and these had variable susceptibility to co-trimoxazole, tetracycline, chloramphenicol and ciprofloxacin. All Gram-positive isolates were sensitive to vancomycin. Of 35 Gram-negative isolates, $10(28.6 \%)$ were resistant to ceftriaxone and $6(17.1 \%)$ to ciprofloxacin. All S. typhimurium isolates were resistant to chloramphenicol, ampicillin and co-trimoxazole, and 4 of $10(40.0 \%)$ had an ESBL phenotype. Two Enterobacter spp., one Klebsiella pneumoniae and one E. coli also had an ESBL phenotype (Table 2). All non-Salmonella Gram-negative isolates were sensitive to gentamicin.

Patients with bloodstream infection received empirical antibiotics with a spectrum broad enough to cover their infecting organism in 20/59 cases (33.9\%). Among patients who had bacteraemia, those who received ineffective empirical antibiotics were more likely to have a poor clinical outcome (OR 8.05; 95\% CI 1.62 39.91; $p=0.04$ ) (Table 3). Of all patients, $597(71.0 \%)$ were prescribed empirical antibiotics by the admitting teams. A total of 50 different empirical antibiotic regimens were used. Penicillin was the single antibiotic most often prescribed. Combination therapy

Table 2. Incidence of specific pathogens causing bloodstream infections, and corresponding antibiogram

\begin{tabular}{|c|c|c|c|c|c|c|c|c|c|c|}
\hline Gram-positive organisms, \% susceptibility & $n$ & PCN & AMP & OX & VAN & TCN & SXT & CAM & CIP & CTX \\
\hline S. aureus & 17 & 0 & 0 & 53 & 100 & 71 & 65 & 76 & 65 & 53 \\
\hline S. pneumoniae & 3 & 67 & 67 & 100 & 100 & 67 & 67 & 100 & 0 & 100 \\
\hline Enterococcus spp. & 2 & 50 & 100 & 0 & 100 & 0 & N/A & 50 & 0 & 0 \\
\hline S. agalactiae & 1 & 100 & 100 & 100 & 100 & 100 & 100 & 100 & 0 & 100 \\
\hline S. pyogenes & 1 & 100 & 100 & 100 & 100 & 100 & 100 & 100 & 0 & 100 \\
\hline Overall susceptibility & 24 & 17 & 25 & 58 & 100 & 67 & 67 & 79 & 46 & 58 \\
\hline Gram-negative organisms, \% susceptibility & $n$ & AMP & TCN & & CAM & SXT & CIP & GEN & CTX & IPM \\
\hline S. typhimurium & 9 & 0 & 67 & & 0 & 0 & 89 & $\mathrm{~N} / \mathrm{A}$ & 55 & 100 \\
\hline S. enteritidis & 1 & 100 & 100 & & 100 & 100 & 100 & $\mathrm{~N} / \mathrm{A}$ & 100 & 100 \\
\hline E. coli & 14 & 21 & 7 & & 43 & 57 & 86 & 100 & 93 & 100 \\
\hline K. pneumoniae & 4 & 0 & 75 & & & 50 & 75 & 100 & 75 & 100 \\
\hline Enterobacter spp. & 2 & 0 & 0 & & 0 & 0 & 50 & 100 & 0 & 100 \\
\hline Citrobacter freundii & 1 & 0 & 0 & & 0 & 0 & 100 & 100 & 100 & 100 \\
\hline Proteus vulgaris & 1 & 100 & 100 & & 100 & 100 & 100 & 100 & 100 & 100 \\
\hline Morganella morganii & 1 & 0 & 0 & & 100 & 0 & 100 & 100 & 100 & 100 \\
\hline Acinetobacter baumanii & 1 & 0 & 0 & & 0 & 0 & 0 & 100 & 0 & 100 \\
\hline Pseudomonas aeruginosa & 1 & 0 & 0 & & 0 & 0 & 100 & 100 & 0 & 100 \\
\hline Overall susceptibility & 35 & 14 & 0 & & 26 & 31 & 83 & 71 & 71 & 100 \\
\hline
\end{tabular}


Table 3. Predictors of poor outcomes in patients with bacteraemia ${ }^{\star}$

\begin{tabular}{|c|c|c|c|c|}
\hline \multirow[b]{2}{*}{$\begin{array}{l}\text { Predictor, } n \text { (total } \\
N=59)^{\star}\end{array}$} & \multicolumn{2}{|c|}{ Outcome } & \multirow[b]{2}{*}{ OR $(95 \% \mathrm{CI})$} & \multirow[b]{2}{*}{$p$-value } \\
\hline & $\begin{array}{c}\text { Unimproved } \\
\text { or died, } n(\%)\end{array}$ & Improved, $n(\%)$ & & \\
\hline $\begin{array}{l}\text { Incorrect empirical } \\
\text { antibiotics }(N=36)\end{array}$ & $17(47.2)$ & $19(52.8)$ & $8.05(1.62-39.91)$ & 0.004 \\
\hline $\begin{array}{l}\text { Correct empirical } \\
\text { antibiotics }(N=20)\end{array}$ & $2(10.0)$ & $18(90.0)$ & $0.12(0.03-0.62)$ & 0.004 \\
\hline $\mathrm{HIV}+(N=45)$ & $11(24.4)$ & $34(75.6)$ & $0.75(0.17-3.43)$ & 0.99 \\
\hline HIV- $(N=10)$ & $3(30.0)$ & $7(70.0)$ & $1.32(0.29-6.02)$ & 0.99 \\
\hline $\begin{array}{l}\mathrm{HIV}+\text { on ARVs } \\
(N=21)\end{array}$ & $6(28.6)$ & $15(71.4)$ & $0.74(0.20-2.78)$ & 0.74 \\
\hline $\begin{array}{l}\text { HIV+ not on ARVs } \\
(N=20)\end{array}$ & $7(35.0)$ & $13(65.0)$ & $1.34(0.36-5.04)$ & 0.74 \\
\hline $\begin{array}{l}\mathrm{SBP} \leq 90 \mathrm{mmHg} \\
(N=10)\end{array}$ & $6(60.0)$ & $4(40.0)$ & $2.0(0.46-8.70)$ & 0.29 \\
\hline $\begin{array}{l}\mathrm{SBP}>90 \mathrm{mmHg} \\
(N=38)\end{array}$ & $12(31.6)$ & $26(68.4)$ & $0.5(0.115-2.17)$ & 0.29 \\
\hline SIRS $(N=40)$ & $10(25.0)$ & $30(75.0)$ & $0.67(0.14-3.17)$ & 0.68 \\
\hline No SIRS $(N=9)$ & $3(33.3)$ & $6(66.7)$ & $1.5(0.32-7.14)$ & 0.68 \\
\hline $\begin{array}{l}\text { Salmonella } \\
\text { bacteraemia }(N=10)\end{array}$ & $2(20.0)$ & $8(80.0)$ & $0.43(0.08-2.25)$ & 0.47 \\
\hline $\begin{array}{l}\text { Non-Salmonella } \\
\text { bacteraemia }(N=46)\end{array}$ & $17(37.0)$ & $29(63.0)$ & $2.34(0.45-12.34)$ & 0.47 \\
\hline $\begin{array}{l}\text { S. aureus } \\
\text { bacteraemia }(N=17)\end{array}$ & $6(35.3)$ & $11(64.7)$ & $1.09(0.33-3.61)$ & 1.0 \\
\hline $\begin{array}{l}\text { Non-S. aureus } \\
\text { bacteraemia }(N=39)\end{array}$ & $13(33.3)$ & $26(66.7)$ & $0.92(0.28-3.03)$ & 1.0 \\
\hline
\end{tabular}

Table 4. Spectrum of antibiotic combinations available at $\mathrm{MCH}$ against bacteraemia isolates

\begin{tabular}{|c|c|c|}
\hline Antibiotic regimen & $\%$ covered & Uncovered organisms \\
\hline CTX alone & 66 & MRSA, ESBL GNRs \\
\hline CTX + GEN & 76 & MRSA \\
\hline CTX + CIP & 75 & $\mathrm{MRSA}^{*} \mathrm{CIP}$-resistant GNRs \\
\hline CTX + GEN + CIP & 81 & $\mathrm{MRSA}^{*} \mathrm{CIP}$-resistant GNRs \\
\hline $\mathrm{VAN}+\mathrm{CTX}$ & 83 & ESBL GNRs \\
\hline VAN+GEN & 83 & Salmonella \\
\hline VAN+GEN + CIP & 98 & CIP-resistant Salmonella \\
\hline VAN + IPM & 100 & None \\
\hline IPM alone & 85 & MRSA \\
\hline
\end{tabular}

with oral antibiotics was common. Hospital doctors adjusted empirical antibiotics before discharge in 153/597 cases (25.6\%). There was no commonly used empirical antibiotic regimen that had $>80 \%$ coverage against all collected isolates. Overall, 20/59 (33.9\%) of all bacterial isolates showed resistance to ceftriaxone, the broadest-spectrum antibiotic commonly available at $\mathrm{MCH}$. The spectrums of several antibiotic regimens potentially available at $\mathrm{MCH}$ are shown in Table 4. It should be noted that vancomycin and imipenem were rarely used by doctors and are not readily available at $\mathrm{MCH}$.
In only $4 / 59$ cases of bloodstream infection (6.8\%) was the diagnosis of bacteraemia or sepsis recorded in the chart at discharge. The most common diagnosis obtained from medical records was tuberculosis, accounting for $>25 \%$ of all patients. Tuberculosis was usually diagnosed empirically; in only $13.5 \%$ of cases was there a positive smear, and cultures were virtually never done. HIV was recorded as the sole diagnosis for $23.1 \%$ of enrolled patients. Eleven patients with bacteraemia were diagnosed as having tuberculosis.

\section{Discussion}

As has been reported at other hospitals in SSA, bacteraemia is a common cause of fever at $\mathrm{MCH}$, accounting for nearly $8 \%$ of all cases of fever in patients newly admitted to the internal medicine wards. That it was not a significant risk factor for in-hospital mortality largely reflects the severity of illness and consequent high mortality rate of patients admitted to $\mathrm{MCH}$. Furthermore, we were not able to follow up patients after discharge, so the 30-day mortality rate for patients with bacteraemia was probably higher. The predominance of $S$. aureus was not surprising, although most studies in the region report NTS to be more common than E. coli as a cause of invasive disease. ${ }^{[1]}$ NTS was the most common isolate from febrile patients during the first period (September 2011 - October 2012), but dropped off significantly in the second period (July 2013 - March 2014), being surpassed by $E$. coli and $S$. aureus. The vector and routes of transmission for NTS in SSA are largely unknown; it is possible that some environmental factor during the time we were collecting specimens affected the NTS incidence. Several factors may account for the low number of Streptococcus pneumoniae, expected to be higher based on other regional reports. ${ }^{[1]}$ Although no patients enrolled in this study were documented to have received antibiotics at $\mathrm{MCH}$ before having their blood cultures drawn, as $\mathrm{MCH}$ is a referral centre, many patients may have received antibiotics at peripheral hospitals before transfer. Antibiotic use is widespread in the community, as in other developing countries, ${ }^{[12]}$ and it may simply be that most patients with invasive $S$. pneumoniae are treated before presenting at $\mathrm{MCH}$. These factors may have decreased the overall rate of bacteraemia in this study, or selected for a sample with a higher resistance rate than that of the community.

Antibiotic resistance was common in the bloodstream pathogens we isolated. Nearly half of the $S$. aureus isolates were MRSA 
and resistant to all first-line antibiotics commonly used at $\mathrm{MCH}$. More than $25 \%$ of all Gram-negative organisms had an ESBL phenotype. S. typhimurium, resistant to ampicillin, chloramphenicol and co-trimoxazole, is commonly reported in the region. ${ }^{[13,14]}$ All $S$. typhimurium isolates described here showed this multidrugresistant phenotype, and 4 of 9 isolates had an ESBL phenotype in addition. This is the first report of invasive ESBL S. typhimurium in Mozambique. Unfortunately, even given this small sample size it appears that there is no simple empirical antibiotic regimen consisting of readily available antibiotics at $\mathrm{MCH}$ that will cover all the most common pathogens causing bacteraemia. Penicillinbased regimens that are commonly used at $\mathrm{MCH}$ are inadequate. Ceftriaxone is used commonly, and was active against $71 \%$ of Gramnegative rods (GNRs) reported here, but is ineffective against MRSA, which accounts for a significant portion of bacteraemia at $\mathrm{MCH}$. Vancomycin was effective against $100 \%$ of Gram-positives collected in this study, but it is relatively expensive and in short supply at $\mathrm{MCH}$, and therefore not recommended for widespread empirical use. However, these results underscore the value of vancomycin, and suggest that clearer local guidelines and an antibiotic stewardship programme at $\mathrm{MCH}$ could help to ensure its judicious use and save lives. Of the other readily available antibiotics, chloramphenicol has the best coverage of the MRSA reported here, but cannot be recommended for empirical use based on these data. The addition of ciprofloxacin to empirical regimens will expand the spectrum to cover most of the ESBL pathogens described here, and will also cover $>50 \%$ of the MRSA, although S. aureus has been shown to acquire resistance to ciprofloxacin rapidly. ${ }^{[15]}$ Gentamicin was effective against all nonSalmonella GNRs, but empirical regimens in patients with suspected bacteraemia at MCH (particularly those with AIDS) must cover NTS, given local epidemiology. All GNRs isolated in the course of this study were susceptible to imipenem, which was not consistently available at MCH during the time of this study. These results support the argument for a well-monitored supply of carbapenem antibiotics. According to these data, if neither vancomycin nor imipenem (or other carbapenem antibiotics) are available, the empirical three-drug regimen offering the broadest spectrum at $\mathrm{MCH}$ is ceftriaxone + gentamicin + ciprofloxacin ( $81 \%$ coverage, Table 3$)$, although this regimen was used infrequently during the time of this study.

There was wide variation in empirical antibiotic regimens prescribed to febrile patients in the medical wards at $\mathrm{MCH}$, and inappropriate empirical choices were linked with bad outcomes. Empirical regimens were infrequently changed during the course of hospitalisation, reflecting an underutilisation of microbiology services at $\mathrm{MCH}$, as well as problems with transmission of information between the laboratory and the wards. Given the rate of antibiotic resistance reported here, it is essential for physicians to maintain a high index of suspicion for bacteraemia, and for them to order blood cultures appropriately in febrile patients. The laboratory must also be able to provide information regarding positive blood cultures and antibiotic susceptibilities promptly, in order to guide subsequent antibiotic adjustment. It was frequently noted during the course of the study that patient follow-up and data completeness were hampered by paper records that were often incomplete, illegible or lost. These problems also affect reporting laboratory information and almost certainly affect clinical care and patient outcomes. Although our study team did not take part in the clinical care of patients, they made every effort to inform doctors when their patients had bacteraemia. A variety of factors such as poorly tracked patient transfers and chart movements, difficulty in contacting doctors charged with care for specific patients, and understaffing in the labs and on the wards meant that this was not always successful. As more hospitals in the developing world move from paper charts to electronic medical records, there is hope that these problems can be minimised and patient outcomes improved.

\section{Conclusion}

Patients admitted to $\mathrm{MCH}$ with bacteraemia have poor outcomes. This study highlights several opportunities for quality improvement, including educating physicians to have a higher index of suspicion for bacteraemia in patients with fever, improving local guidelines for empirical antibiotic therapy and improving communication between the microbiology laboratory and the clinicians, as well as increasing the supply of some key antibiotics such as vancomycin and imipenem.

Funding. This research was supported in part by the University of California Center for AIDS Research, a National Institutes of Healthfunded programme (P30 AI036214) supported by the following $\mathrm{NIH}$ Institutes and Centers: NIAID, NCI, NIMH, NIDA, NICHD, NHLBI, NIA, NIGMS and NIDDK, and in part by the Fogarty International Center by 1R24TW008908-01: The Eduardo Mondlane Medical Education Partnership. Additionally, the project described was partially supported by the National Institutes of Health, Grant KL2RR031978.

\footnotetext{
References

1. Reddy EA, Shaw AV, Crump JA. Community-acquired bloodstream infections in Africa: A systematic review and meta-analysis. Lancet Infect Dis 2010;10(6):417-432. [10.1016/S1473-3099(10)70072-4]

. Iroha IR, Esimone CO, Neumann S, et al. First description of Escherichia coli producing CTX-M15-extended spectrum beta lactamase (ESBL) in out-patients from south eastern Nigeria. Ann Clin Microbiol Antimicrob 2012;11:19. [http://dx.doi.org/10.1186/1476-0711-11-19]

3. Schaumburg $\mathrm{F}$ Alabi A Kokou C et al High burden of extended-spectum Schaumburg F, Alabi A, Kokou C, et al. High burden of extended-spectrum beta-lactamase-producing Enterobacteriaceae in Gabon. J Antimicrob Chemother 2013;68(9):2140-2143. [http://dx.doi.
org/10.1093/jac/dkt164]

4. Drain PK, Losina E, Parker G, et al. Risk factors for late-stage HIV disease presentation at initial HIV diagnosis in Durban, South Africa. PLoS One 2013;8:e55305. [http://dx.doi.org/10.1371/journal. pone.0055305

5. Micek MA, Gimbel-Sherr K, Baptista AJ, et al. Loss to follow-up of adults in public HIV care systems in central Mozambique: Identifying obstacles to treatment. J Acquir Immune Defic Syndr 2009;52(3):397405. [http://dx.doi.org/10.1097/QAI.0b013e3181ab73e2]

6. Bedell RA, Anderson ST, van Lettow M, et al. High prevalence of tuberculosis and serious bloodstrean infections in ambulatory individuals presenting for antiretroviral therapy in Malawi. PLoS One 2012;7:e39347. [http://dx.doi.org/10.1371/journal.pone.0039347]

7. Mandomando I, Sigauque B, Morais L, et al. Antimicrobial drug resistance trends of bacteremia isolates in a rural hospital in southern Mozambique. Am J Trop Med Hyg 2010;83(1):152-157. [http:// dx.doi.org/10.4269/aitmh.2010.09-0578

8. Moon TD, Silva WP, Buene M, et al. Bacteremia as a cause of fever in ambulatory, HIV-infected Mozambican adults: Results and policy implications from a prospective observational study. PLoS One 2013;8:e83591. [http://dx.doi.org/10.1371/journal.pone.0083591]

MEPI Program Paspectives from Glass NI, Razak MH, Said M. The importance of research in the MEPI Progrant. Perspectives from the National Institutes of 0000351

10. Noormahomed EV, Mocumbi AO, Preziosi M, et al. Strengthening research capacity through the medical education partnership initiative: The Mozambique experience. Hum Resour Health 2013;11:62. [http://dx.doi.org/10.1186/1478-4491-11-62]

11. Cockerill FR, Wikler MA, Alder, J, et al. CLSI Performance Standards for Antimicrobial Susceptibility Testing: Twenty-second Informational Supplement. Wayne, PA: Clinical and Laboratory Standard Institute, 2012

2. Van Boeckel TP, Gandra S, Ashok A, et al. Global antibiotic consumption 2000 to 2010: An analysis of national pharmaceutical sales data. Lancet Infect Dis 2014;14(8):742-750. [http://dx.doi.org/10.1016/ S1473-3099(14)70780-7]

13. Okoro CK, Kingsley RA, Connor TR, et al. Intracontinental spread of human invasive Salmonell typhimurium pathovariants in sub-Saharan Africa. Nat Genet 2012;44(11):1215-1221. [http://dx.doi. org/10.1038/ng.2423]

14. Kingsley RA, Msefula CL, Thomson NR, et al. Epidemic multiple drug resistant Salmonell typhimurium causing invasive disease in sub-Saharan Africa have a distinct genotype. Genome Res 2009:19(12):2279-2287. [http///dx.doi org/10.1101/gr.091017.109]

15. McGowan JEJ. Abrupt changes in antibiotic resistance. J Hosp Infect 1991;18(Suppl A):202-210
} 